OPEN

SUBJECT AREAS:

DIABETES

COMPLICATIONS

METABOLIC SYNDROME

Received

12 March 2014

Accepted

16 June 2014

Published

3 July 2014

Correspondence and requests for materials should be addressed to X.Q.D.

(xiaoqun_dong@mail. uri.edu)

\title{
Clinical implication of changes in serum adiponectin in patients with hepatogenic diabetes
}

\author{
Zhongying Bao', Xiaodong Yuan', Shuhong Duan' \& Xiaoqun Dong²
}

\begin{abstract}
'Department of Infectious Diseases, Beijing Shijitan Hospital, Capital Medical University (The $9^{\text {th }}$ affiliated hospital of Peking University), Beijing, 100038, P.R. China, ${ }^{2}$ Department of Biomedical and Pharmaceutical Sciences, College of Pharmacy, University
\end{abstract} of Rhode Island, Kingston, RI 02881, USA.

Adiponectin is a protein hormone that modulates glucose metabolism and fatty acid oxidation. We explored the clinical implication of serum adiponectin in hepatogenic diabetes. Serum adiponectin levels were determined using enzyme-linked immunochemistry assay in 78 individuals including 19 hepatogenic diabetes, 20 type 2 diabetes (T2D), 20 chronic liver disease and 19 healthy controls. Cases and controls were matched by gender and body mass index (BMI). There is no difference in serum adiponectin levels among hepatogenic diabetic, T2D and healthy control groups. The levels of adiponectin are highest in chronic liver disease and lowest in T2D. Insulin levels are highest in hepatic diabetics and lowest in T2D. Hepatic diabetics have the lowest insulin sensitivity index (ISI). Serum adiponectin levels were negatively correlated with triglycerides and total cholesterol in T2D. Serum adiponectin is significantly increased in chronic liver disease, but lacks association with hepatogenic diabetes.

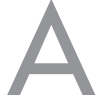

diponectin is an adipocyte-derived hormone encoded by $A D I P O Q$ gene. Several genome-wide association studies have mapped $A D I P O Q$ gene located on chromosome $3 \mathrm{q} 27$ as a susceptibility locus for type 2 diabetes (T2D) and metabolic syndrome $e^{1}$. Decreased expression of adiponectin may confer insulin resistance in murine models of altered insulin sensitivity ${ }^{1}$. Adiponectin is proposed to reverse insulin resistance by decreasing triglyceride content in muscle and liver in obese mice ${ }^{1}$, which results from increased expression of molecules involved in fatty-acid combustion and energy dissipation in muscle ${ }^{1-3}$. In addition, insulin resistance in lipoatrophic mice was completely reversed by the combination of physiological doses of adiponectin and leptin, but only partially by either adiponectin or leptin alone ${ }^{1}$. Thus the replenishment of adiponectin may provide a novel treatment modality for insulin resistance and $\mathrm{T}^{1} \mathrm{D}^{1}$.

Accumulating evidence has shown that adiponectin is involved in multiple important biological functions such as regulating glucose transporter, enhancing insulin sensitivity, and attenuating inflammation ${ }^{4}$. Notably, chronic hepatitis has been shown to cause changes in adiponectin levels ${ }^{5,6}$. However, there are very few studies on the change in adiponectin levels in patients with hepatogenic diabetes. Therefore, we compared the content of the plasma adiponectin of healthy controls to patients with hepatogenic diabetes, chronic liver disease and T2D. We tested whether the changes in adiponectin levels correlates with the above-mentioned diseases and evaluated the value of adiponectin as an early diagnostic marker for hepatogenic diabetes.

\section{Results}

The differences in age, waist, body mass index (BMI), serum adiponectin, fasting glucose, fasting insulin, total cholesterol, triglycerides, and insulin sensitivity index (ISI) were compared among the four groups (Table 1).

Plasma adiponectin levels. A significant difference in plasma adiponectin levels was observed among the four groups $(P<0.01)$. Patients with chronic liver disease had the highest, followed by the healthy control and hepatogenous diabetic group, T2D had the lowest level of adiponectin.

Fasting glucose. There was a significant difference in fasting glucose levels among the four groups $(P<0.01)$. Compared to healthy controls or patients with chronic liver disease, T2D and hepatogenous diabetics had increased fasting glucose levels $(P<0.05)$. 
Table 1 | Clinical indicator (mean \pm SD) in study population

\begin{tabular}{lcccc} 
& \multicolumn{3}{c}{ Groups } \\
\cline { 2 - 5 } & Healthy control $(\mathrm{n}=19)$ & Hepatogenic diabetes $(\mathrm{n}=19)$ & Type II diabetes $(\mathrm{n}=20)$ & Chronic liver disease $(\mathrm{n}=20)$ \\
\hline Age & $48 \pm 4.5$ & $53 \pm 8.6$ & $57 \pm 6.2$ & $51 \pm 4.1$ \\
Waist $(\mathrm{WC})$ & $82.9 \pm 5.12$ & $84.69 \pm 6.48$ & $86.1 \pm 7.07$ & $83.28 \pm 4.34$ \\
$\mathrm{BMI}$ & $21.7 \pm 3.3$ & $22.7 \pm 2.6$ & $23.3 \pm 1.8$ & $22.0 \pm 1.9$ \\
Adiponectin $(\mu \mathrm{g} / \mathrm{mL})$ & $7.16 \pm 3.45$ & $5.56 \pm 3.04$ & $2.84 \pm 0.85$ & $10.24 \pm 6.23^{*}$ \\
Glucose $(\mathrm{mg} / \mathrm{dL})$ & $3.3 \pm 2.5^{\Delta}$ & $8.8 \pm 2.4$ & $9.6 \pm 4.0^{\&}$ & $4.2 \pm 2.0^{*}$ \\
Insulin $(\mu \mathrm{lU} / \mathrm{mL})$ & $9.8 \pm 3.1^{\Delta}$ & $15.5 \pm 4.1$ & $8.2 \pm 3.1^{\&}$ & $10.6 \pm 4.4^{*}$ \\
Triglycerides $(\mathrm{mmol} / \mathrm{L})$ & $1.22 \pm 0.29$ & $1.06 \pm 0.64$ & $2.04 \pm 0.51^{\&}$ & $1.13 \pm 0.30$ \\
Total cholesterol $(\mathrm{mmol} / \mathrm{L})$ & $3.61 \pm 0.46$ & $3.32 \pm 0.85$ & $5.53 \pm 1.03^{\&}$ & $3.28 \pm 0.88$ \\
Insulin sensitivity index & $-1.66 \pm 0.14^{\Delta}$ & $-2.04 \pm 0.17$ & $-1.83 \pm 0.24^{\&}$ & $-1.68 \pm 0.21^{*}$ \\
\hline$* P<0.05$, comparison between hepatogenic diabetes and chronic liver disease. & & & \\
AP $<0.05$, comparison between hepatogenic diabetes and healthy control. & & & \\
\&P $<0.05$, comparison between hepatogenic diabetes and type 2 diabetes. & & & \\
\hline
\end{tabular}

Fasting insulin. We found a significant difference in fasting insulin levels among four groups were $(P<0.01)$. Higher levels of fasting insulin were detected in T2D and hepatogenous diabetics than in healthy controls or chronic liver disease $(P<0.01)$. T2D patients had even higher insulin levels than hepatogenic diabetics $(P<0.01)$.

Triglycerides. T2D had higher triglycerides than other subjects $(P<$ 0.05).

Total cholesterol. T2D showed increased total cholesterol levels than other subjects $(\mathrm{P}<0.01)$.

Insulin sensitivity Index (ISI). Patients with hepatogenous diabetes presented significantly decreased ISI compared with other subjects $(P<0.01)$.

Correlation of serum adiponectin with other clinical indicators. Serum adiponectin levels negatively correlated with triglycerides and total cholesterol levels in all subjects as demonstrated in Table 2. Compared with healthy control, serum adiponectin levels in T2D negatively correlated with triglycerides and total cholesterol levels, but positively correlated with ISI $(P<0.01)$ as illustrated in Table 3.

\section{Discussion}

Recent studies suggest that adiponectin can significantly improve insulin resistance and enhance peripheral tissue sensitivity to insulin, which may serve as a target of T2D treatment. Hyperinsulinemia and insulin resistance are the main features of hepatogenic diabetes. The role of adiponectin in hepatic diabetes is unclear. We are interested in whether adiponectin is a diagnostic indicator and a potential therapeutic target of hepatogenic diabetes. Insulin resistance has been proposed as one of the most important causes of liver-induced diabetes $^{7}$. A precise method to determine insulin resistance was the insulin clamp technique; however, the high expense has limited its clinical application. Thus amendments have been proposed to improve the minimal model ${ }^{8}$. Insulin sensitivity index (ISI) may replace the insulin clamp technique, as an alternative marker to reflect insulin resistance of hepatogenic diabetes ${ }^{9}$. Consistently, we found ISI in hepatogenic diabetics was decreased significantly comparing with other groups, suggesting the presence of insulin resistance $^{8}$. A recent study on rhesus monkeys observed that circulating concentrations of the adiponectin are decreased in parallel with reduced insulin sensitivity during the progression to $\mathrm{T}_{2} \mathrm{D}^{10}$.

\begin{tabular}{|lccc|}
\hline \multicolumn{3}{|c|}{ Table 2 | } & Correlation of serum adiponectin with clinical indicators \\
& Triglycerides & Total cholesterol & Insulin sensitivity index \\
\hline R values & -2.79 & -1.6 & 0.032 \\
P values & 0.013 & 0.043 & 0.78 \\
\hline
\end{tabular}

Adiponectin levels were gradually reduced with the development of insulin resistance. In our study, patients with T2D had relatively low levels of baseline insulin compared to healthy controls. We suggest that some patients had low levels of insulin due to dysfunction in secretion or delayed peak at diagnosis.

Hepatogenous diabetes develops in chronic liver disease; especially liver cirrhosis and sex hormone metabolism disorders, where estrogen levels are elevated but androgen are relatively low, resulting in elevated levels of adiponectin. Interestingly, serum adiponectin levels in mice presented gender dimorphism, where the females had higher adiponectin than the males ${ }^{11}$. Adiponectin receptor 1 (AdipoR1) and 2 (AdipoR2) have been implicated in adiponectin hoist caused by hepatic diabetes ${ }^{12}$. Human AdipoR1 was expressed in skeletal muscle and AdipoR2 mainly in the liver. Hepatogenic diabetics may have reduced expression of AdipoRs and thus elevated serum adiponectin due to the destruction and dysfunction of liver cells $^{5,13}$

Adiponectin can be produced by human hepatoma HepG2 cells after treatment with IL- 6 or damaged hepatocytes in response to $\mathrm{CCl}_{4}$-induced injury in mice ${ }^{14}$. Adiponectin levels were elevated during the progression from chronic hepatitis B to liver fibrosis, which declined in response to antiviral therapy ${ }^{15}$. Serum adiponectin levels were significantly increased in liver cirrhosis, associated with the reaction of active inflammation in chronic liver disease ${ }^{16}$.

Adiponectin negatively regulates fatty acid oxidation. We found that the level of triglyceride and total cholesterol were lower in chronic liver disease and hepatogenic diabetes, but higher in T2D. We observed increased insulin levels but decreased insulin sensitivity index in hepatogenic diabetes, which are consistent with a previous study ${ }^{10}$. Another study found no association between adiponectin levels and insulin resistance of varying degrees of liver cirrhosis ${ }^{16}$. In our study, serum adiponectin in healthy controls and T2D negatively correlated with BMI, triglycerides and cholesterol, but positively correlated with insulin sensitivity index.

Hepatogenic diabetics often accompany with varying degrees of liver inflammation and metabolic disorders, which led to the changes in serum adiponectin levels. Although many studies suggest that decreased serum adiponectin levels closely related with T2D, it may not serve as a diagnostic marker for hepatogenic diabetes. Accumulating evidence indicates that dynamic measurements of adiponectin levels may predict hepatogenous diabetes. Mice feed

Table 3 | Correlation of serum adiponectin with other indicators in healthy controls and type II diabetics

\begin{tabular}{lccc} 
& Triglycerides & Total cholesterol & Insulin sensitivity index \\
\hline$R$ values & -0.485 & -7.11 & 0.391 \\
$P$ values & 0.001 & $<0.001$ & 0.01 \\
\hline
\end{tabular}


by high-fat and high-sugar diet can lose weight and present reduced glucose, fatty acids, triglycerides and insulin resistance when treated with the globular domain of adiponectin ${ }^{17}$. Additionally, adiponectin could reduce glucose levels of murine type I diabetes by reducing hepatic glucose output ${ }^{18}$. In our study, adiponectin levels were lower in hepatic diabetics than healthy controls and chronic liver disease. Hepatogenous diabetes may benefit from exogenous adiponectin. The limitations of this study include a small sample size and the absence of other clinical indicators such as tumor necrosis factor. Our findings need to be expanded in a large patient population. The metabolic characteristics and mechanisms of serum adiponectin in hepatic diabetes need further investigation.

\section{Methods}

Patient population. A total of 78 male subjects were recruited at Beijing Shijitan Hospital including 19 cases of hepatogenic diabetes, 20 cases of T2D, 20 cases of chronic liver disease and 19 healthy controls. Among hepatic diabetics (mean age 53 \pm 8.6 years, range $42-71$ years), 17 cases were hepatitis B virus-related and 2 cases were hepatitis $C$ virus-related. T2D patients were newly diagnosed (mean age $57 \pm 6.2$ years, range $37-76$ years). Among the 20 patients with chronic liver disease (mean age $51 \pm 4.1$ years, range $42-67$ years), 17 cases were hepatitis B virus-related and 3 cases were hepatitis $C$ virus-related. The healthy control group consisted of 19 individuals (mean age $48 \pm 4.5$ years, range $37-52$ years) with normal blood pressure, blood lipids, blood glucose levels and no previous history of chronic hepatitis. Each subject had a body mass index (BMI) less than 25 according to the Obesity Guidelines for Asia-Pacific region. Hypertensive patients were excluded from the study. Informed consent was obtained from all subjects. This study was approved by the institutional review board of Beijing Shijitan Hospital Affiliated to Capital Medical University (The 9th affiliated hospital of Peking University) and was carried out in accordance with established national and institutional ethical guidelines regarding the involvement of human subjects and the use of human tissues for research.

Diagnostic, inclusion and exclusion criteria. The diagnosis of T2D is based on the Diagnosis and Classification of Diabetes Mellitus of American Diabetes Association ${ }^{19}$. Diagnosis of chronic viral hepatitis and liver cirrhosis was based on American Association for the Study of Liver Diseases (AASLD) Practice Guideline in $2004^{20}$. The diagnostic criteria for hepatogenic diabetes include $\left.{ }^{8}: 1\right)$ Chronic liver disease occurred before the diabetes; 2) The patient's liver function was abnormal; 3) Elevated glucose was consistent with the deterioration of liver function; 4) Symptoms of diabetes were mild or absent and without the complications of diabetes; 5) Fasting plasma glucose $\geq 7.0 \mathrm{mmol} / \mathrm{L}$ or normal, but the 2-hour postprandial blood glucose $\geq 11.1 \mathrm{mmol} / \mathrm{L}$; oral glucose tolerance test (OGTT) or fasting blood glucose was normal or mildly elevated but postprandial glucose $\geq 11.1 \mathrm{mmol} / \mathrm{L} ; 6$ ) Insulin release test showed high levels of fasting plasma insulin and postprandial insulin response delay; C-peptide/insulin ratio was decreased; 7) Patients had no family history of diabetes. Patients with secondary hyperglycemia caused by the pituitary, adrenal, pancreas, and thyroid disease were exclude. Patients with glucose metabolism disorder caused by drugs such as diuretics, corticosteroids, antihypertensive drugs, and contraceptives were excluded.

Acquisition and processing of specimens. All subjects were fasted for 12-14 hours, and fasting venous blood $5 \mathrm{ml}$ were collected in the next morning. Specimens were centrifuged at $3500 \mathrm{r} / \mathrm{min}$ for 10 minutes; serum was separated and kept at $-80^{\circ} \mathrm{C}$.

Determination of adiponectin. Adiponectin was demined using Enzyme-linked immunochemistry assay (ELISA) according to the manufacturer's instruction. The ELISA kit was purchased from Jingmei Biotech Co Ltd. The data was analyzed using the MRX-automatic production fluorescence microplate reader (Dynex

Technologies, USA).

Measurement of Glucose, triglycerides, and total cholesterol. Concentrations of blood glucose, triglycerides, and total cholesterol applied Hitachi 7170 automatic biochemical analyzer.

Determination of fasting insulin. Fasting insulin was measured using chemiluminescence detection.

ISI (insulin sensitive index). ISI was calculated as the natural logarithm of the reciprocal of product fasting blood glucose and fasting insulin.

Statistical analysis. Statistical analysis was performed using SPSS software (version 11.5). The measurement data were expressed as mean \pm standard deviation (SD). For the data with normal distribution, the differences between groups were examined by pairwise comparisons; otherwise, data with skewed distribution were first transformed to log-normal distribution. Association between different indicators was evaluated by Pearson correlation. A $P<0.05$ was considered as statistically significant.
1. Yamauchi, T. et al. The fat-derived hormone adiponectin reverses insulin resistance associated with both lipoatrophy and obesity. Nat. Med. 7, 941-946 (2001).

2. Vionnet, N. et al. Genomewide search for type 2 diabetes-susceptibility genes in French whites: evidence for a novel susceptibility locus for early-onset diabetes on chromosome 3q27-qter and independent replication of a type 2-diabetes locus on chromosome 1q21-q24. Am. J. Hum Genet. 67, 1470-1480 (2000).

3. Kissebah, A. H. et al. Quantitative trait loci on chromosomes 3 and 17 influence phenotypes of the metabolic syndrome. Proc Natl Acad Sci U S A. 97, 14478-14483 (2000).

4. Flier, J. S. Diabetes. The missing link with obesity? Nature. 409, 292-293 (2001)

5. Li, X. et al. Adiponectin and its receptors in chronic hepatitis B patients with metabolic syndrome in China. Hepatogastroenterology 59, 1735-1743 (2012).

6. Khattab, M. A. et al. Association of serum adipocytokines with insulin resistance and liver injury in patients with chronic hepatitis $\mathrm{C}$ genotype 4 . J Clin Gastroenterol. 46, 871-879 (2012).

7. Garcia-Compean, D. et al. The prevalence and clinical characteristics of glucose metabolism disorders in patients with liver cirrhosis. A prospective study. Ann Hepatol. 11, 240-248 (2012).

8. Gundling, F., Schumm-Draeger, P. M. \& Schepp, W. [Hepatogenous diabetes diagnostics and treatment]. Z Gastroenterol. 47, 436-45 (2009).

9. Perseghin, G. et al. Contribution of reduced insulin sensitivity and secretion to the pathogenesis of hepatogenous diabetes: effect of liver transplantation. Hepatology. 31, 694-703 (2000)

10. Hotta, K. et al. Circulating concentrations of the adipocyte protein adiponectin are decreased in parallel with reduced insulin sensitivity during the progression to type 2 diabetes in rhesus monkeys. Diabetes. 50, 1126-1133 (2001).

11. Pajvani, U. B. et al. Structure-function studies of the adipocyte-secreted hormone Acrp30/adiponectin. Implications fpr metabolic regulation and bioactivity. J. Biol. Chem. 278, 9073-9085 (2003).

12. Yamauchi, T. et al. Targeted disruption of AdipoR1 and AdipoR2 causes abrogation of adiponectin binding and metabolic actions. Nat. Med. 13, 332-339 (2007).

13. Wu, D. et al. Adiponectin and its receptors in chronic hepatitis B patients with steatosis in china. Hepat. Mon. 13, e6065 (2013).

14. Yoda-Murakami, M. et al. Change in expression of GBP28/adiponectin in carbon tetrachloride-administrated mouse liver. Biochem. Biophys. Res. Commun. 285, 372-327 (2001).

15. Hui, C. K. et al. Serum adiponectin is increased in advancing liver fibrosis and declines with reduction in fibrosis in chronic hepatitis B. J. Hepatol. 47, 191-202 (2007).

16. Kaser, S. et al. Circulating adiponectin reflects severity of liver disease but not insulin sensitivity in liver cirrhosis. J. Intern. Med. 258, 274-80 (2005).

17. Fruebis, J. et al. Proteolytic cleavage product of $30-\mathrm{kDa}$ adipocyte complementrelated protein increases fatty acid oxidation in muscle and causes weight loss in mice. Proc Natl Acad Sci U S A. 98, 2005-2010 (2001).

18. Berg, A. H., Combs, T. P., Du, X., Brownlee, M. \& Scherer, P. E. The adipocytesecreted protein Acrp30 enhances hepatic insulin action. Nat. Med. 7, 947-953 (2001).

19. American Diabetes, A. Diagnosis and classification of diabetes mellitus. Diabetes Care 37 Suppl 1, S81-90 (2014).

20. Hendricks, D. A. Corrections to the AASLD Practice Guideline. Hepatology 40, 268 (2004)

\section{Acknowledgments}

This study was supported by National Science Foundation of China (Grant Number 81100366 to ZB).

\section{Author contributions}

Z.Y.B. was responsible for experimental design and supervised the study. Z.Y.B., X.D.Y. and S.H.D. developed methodology. X.D.Y. and S.H.D. carried out the experiments. Z.Y.B. performed data analysis and prepared the figures and tables. Z.Y.B., X.D.Y., S.H.D. and X.Q.D. wrote, reviewed, and revised the manuscript.

\section{Additional information}

Competing financial interests: The authors declare no competing financial interests.

How to cite this article: Bao, Z.Y., Yuan, X.D., Duan, S.H. \& Dong, X.Q. Clinical implication of changes in serum adiponectin in patients with hepatogenic diabetes. Sci. Rep. 4, 5560; DOI:10.1038/srep05560 (2014).

This work is licensed under a Creative Commons Attribution-NonCommercialNoDerivs 4.0 International License. The images or other third party material in this article are included in the article's Creative Commons license, unless indicated otherwise in the credit line; if the material is not included under the Creative Commons license, users will need to obtain permission from the license holder in order to reproduce the material. To view a copy of this license, visit http:// creativecommons.org/licenses/by-nc-nd/4.0/ 